\title{
How Soar Agent Learns: Episodic Memory
}

\author{
Nitin Bansal \\ Computer Science, Research Scholar \\ Mewar University \\ Chhitorgarh, Rajasthan
}

\author{
S. Srinivasan, $\mathrm{PhD}$ \\ HOD, CSA Department \\ PDM University \\ Bahadurgarh, Haryana
}

\begin{abstract}
The main objective of this paper is to make the agent learn from its experiences in order to improve its intelligence. Learning is performed with the help of episodic memory which store the previous experiences in the form of episodes in order to solve the problem. With the help of the implementation in soar of an example, this paper presents the improvement in intelligence. In this paper episodes (previous experiences) which are in the form of WME (working memory elements) work to improve the intelligence. Objective of this paper is to show that learning always improves performance i.e. as agent learns more, the performance will improve.
\end{abstract}

\section{General Terms}

Cognitive Architecture, Artificial Intelligence, Cognitive Capabilities, Long-Term Memory, Short-term Memory.

\section{Keywords}

Soar, Working Memory, Episodic Memory, VisualSoar, epmem, Cues, Working Memory Elements.

\section{INTRODUCTION}

Cognitive architecture's aim is the development of artificial agents that support the same capabilities as that of the human mind [1]. From the various cognitive architectures available we take soar into consideration. Soar is a general cognitive architecture for developing systems that exhibit intelligent behavior [5]. Soar stands for State, Operator and Result because all problem solving in Soar is viewed as a search through a problem space in which you apply an operator to a state to get a result. It support all the capabilities required of a general intelligent agent [11]

One advantage that humans have over current artificial intelligence (AI) systems is a personal history of specific events from their past that they can draw upon to improve their learning and decision making. Episodic memory of soar does the same work. It was first described in depth by Endel Tulving $(1983,2002)$ [6].

\section{EPISODIC MEMORY}

Episodic memory contains memories of what was experienced over time, episodic memory is distinguished by the fact that it is task-independent and thus available for every problem, providing a memory of experience not available from other mechanisms. In Soar, episodic memory includes specific instances of the structures that occur in working memory at the same time, providing the ability to remember the context of past experiences as well as the temporal relationships between experiences [14].It contains temporally ordered "snapshots" of working memory. An episode contains agent's current state, which includes perception, motor commands, and internal data structures. Episodic learning remembers events and history that are embedded in experience. The ability to remember where you have been, what you have sensed and what actions you have taken in various situations provides a knowledge base of information that is invaluable for acting in the present. Knowing your personal history facilitates your ability to perform several cognitive capabilities in the context of sensing, reasoning and learning.

\section{WORKING MECHANISM OF EPISODIC MEMORY}

When an agent attempts to retrieve an episode, it creates a memory cue in the "cue" area. In this paper we will concentrate on the matching procedure of WMEs with the episodic memory contents [2].Episodic memory stores the entire snapshot of working memory images which encodes the agent's experience. What is retrieved from episodic memory is the best partial match. Only one episode is retrieved at a time. An episode is retrieved by the deliberate creation of a cue, which is a partial specification of working memory in a special buffer. Once a cue is created, the best partial match is found (biased by decency and working memory activation) and retrieved into a separate working memory buffer (to avoid confusion between a memory and the current situation). The next episode can also be retrieved, providing the ability to replay an experience as a sequence of retrieved episodes [2].

Once a cue is created, episodic memory finds the best partial match and recreates it in a separate working memory buffer (to avoid confusion between a memory and the current situation). The agent can also use cues that move forward or backward in time from a retrieved episode, providing the ability to replay an experience as a sequence of retrieved episodes. We have demonstrated that when episodic memory is embedded in Soar, it enables many advanced cognitive capabilities such as virtual sensing, internal simulation and prediction, learning action models, and retrospective reasoning and learning [9].

\section{EPISODIC MEMORY IN SOAR}

Episodic memory in cognitive architectures allows intelligent agents to query their past experiences to influence decision making. Episodic memory in the Soar cognitive architecture must efficiently encode, store, search and reconstruct episodes as snapshots of short term working memory 


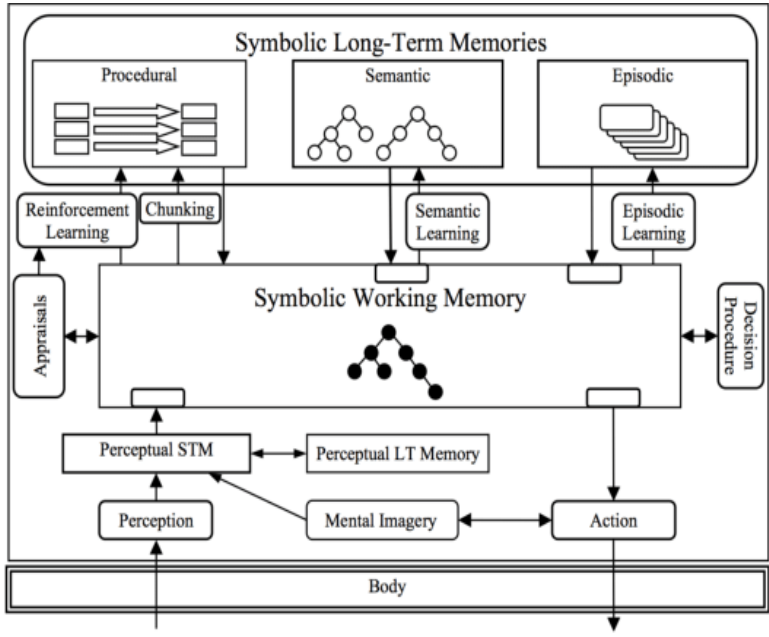

Fig 1: Episodic memory in soar

All decisions are made through the combination of relevant knowledge at run-time. In Soar, every decision is based on the current interpretation of sensory data, the contents of working memory created by prior problem solving, and any relevant knowledge retrieved from long-term memory [10]. Decisions are never precompiled into uninterruptible sequences.

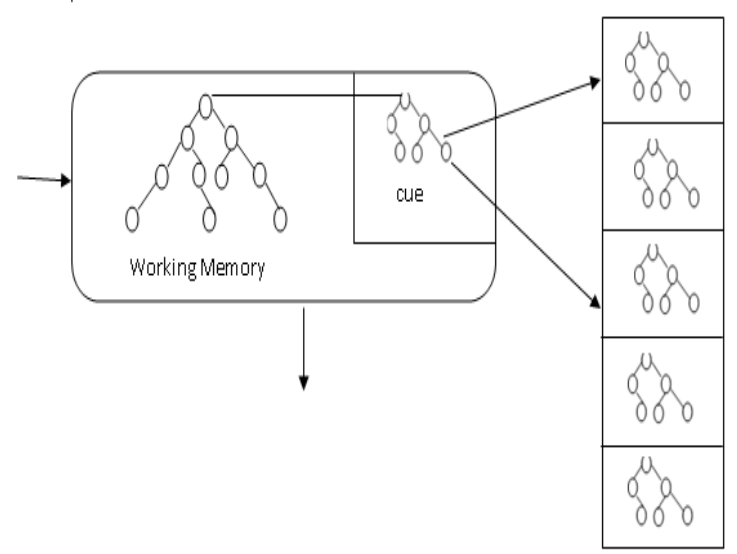

Episodic Memory: Contains various episodes

Fig 2: cues in working memory and episodic memory

An agent's information about its current situation is called its "state", which is maintained in working memory at current time. An episode consists of an agent's state including input, internal data structure and output [2].

Episodic memory is processed into following phases:

- Encoding

- Storage

- Retrieval

\subsection{Encoding}

In this phase we initiation encoding i.e. When is an episode encoded? What are the contents of an episode? (Episode determination) and decides is a subset of features in the stored episodes selected for matching during retrieval also called Feature selection.

\subsection{Storage}

Storage phase is to decide about the storage of the Episode structure i.e. what is the structure of the episodic memory to be stored? And the episodic store change over time, such as through forgetting or generalization which is considered as Episode dynamics?

\subsection{Retrieval}

This phase is the final phase which will retrieve the matched episodes and therefore perform learning by agent. It does Retrieval initiation which will decide when is an episode retrieved? After this, Cue determination is performed i.e. How is the cue specified? Then we does Retrieval to a given cue, which episode is retrieved? And finally Retrieved episode is represented after an episode is retrieved and will decide how is it represented in the agent?

This phase has also Retrieve meta-data to represent is there additional meta-data about the episode and its match to the cue?

\section{EPISODIC MEMORY INTEGRATION}

In our implementation, snapshots of working memory are captured and automatically stored in episodic memory [7]. Everything in working memory is stored in the episode except for WMEs with very low activation (indicating they were not recently created or tested). Episodic memory is queried by an operator creating a structure in the cue area of working memory. The episode that is the best match to the cue is found and deposited in the retrieved area of working memory.

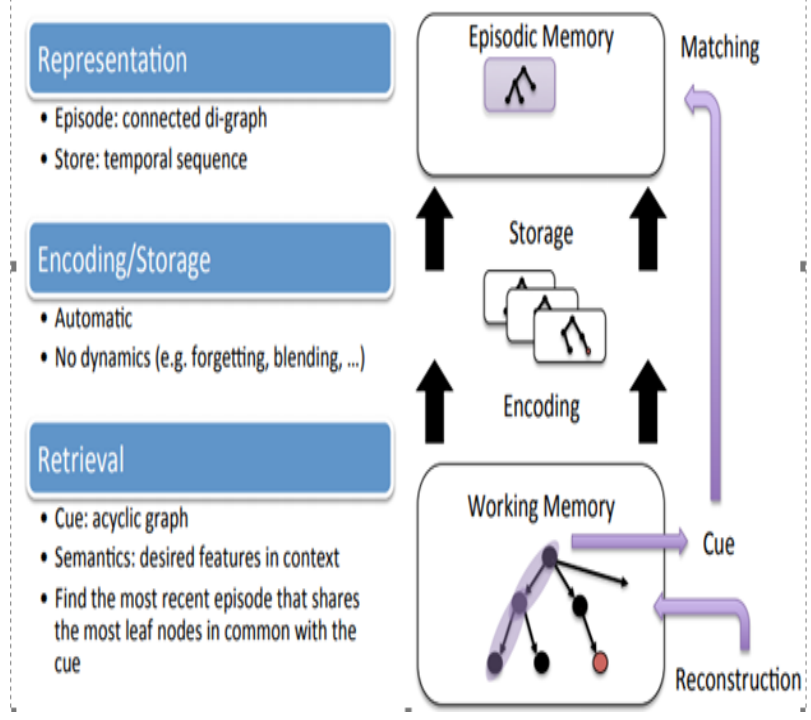

Fig 3: episodic memory integration

\section{EPISODIC MEMORY IMPLEMENTATION IN SOAR}

Episodic memory (EpMem) in Soar is a mechanism that automatically captures, stores and temporally indexes agent state and supports a content-addressable agent interface to retrieve this autobiographical prior experience [4].

\subsection{Capturing an episode and viewing the contents of the memory}

Open the Soar Debugger. Then, execute the following command:

epmem --set trigger dc

epmem --set learning on 
watch -epmem

Click the "Step" button twice and see the

following message

NEW EPISODE: 1

Soar Debugger in lava - 50arl

File Edit Print Commands DebugLevel Layout Agents Kernel Help

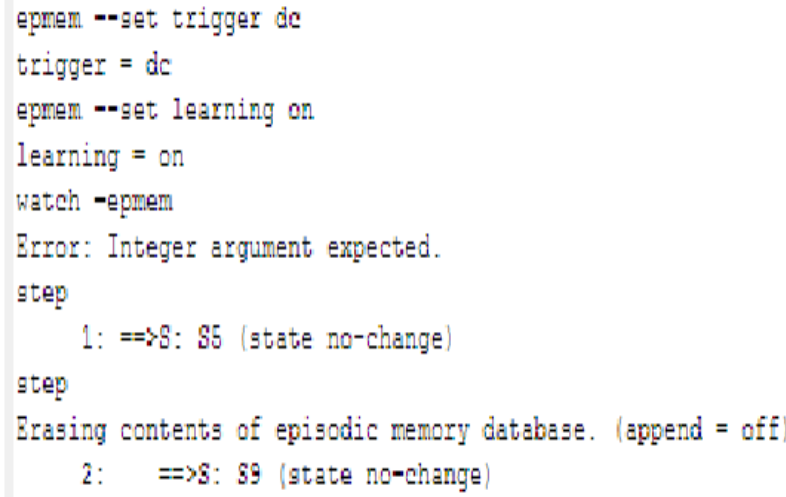

Fig 4: Soar debugger window for watching episode

\subsection{View the contents of episodic memory}

To view the contents of episodic memory we use command:

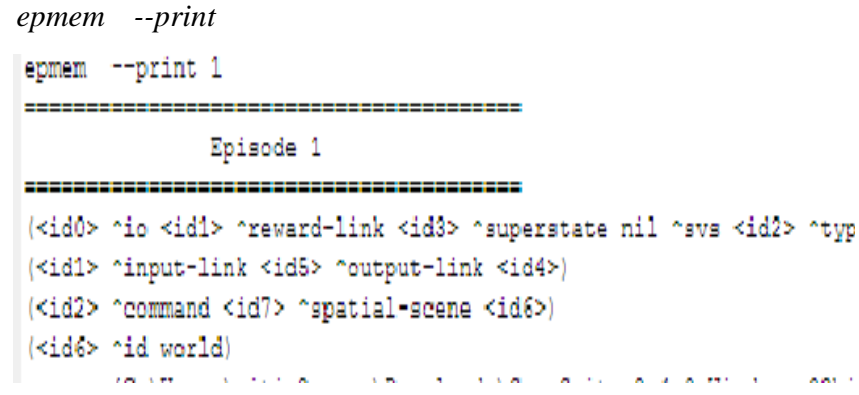

Fig 5: Soar debugger window with contents of episodic memory

\subsection{Episodic Storage}

Episodic storage is automatic and captures the top state of the agent's working memory. To enable storage, episodic memory must be enabled. By default, all learning mechanisms in Soar are disabled. To enable episodic memory, issue the following command:

$$
\text { epmem --set learning on }
$$

By default, episodic memory stores new episodes whenever a WME is added to working memory that has the output-link as its identifier. However, Soar also supports storing episodes each decision cycle ("dc"), which is enabled using the following command:

$$
\text { epmem --set trigger } d c
$$

The next important parameter is the phase during which episodic memory stores episodes.for this command is:

$$
\text { epmem --set phase selection }
$$

epmem --set phase selection

phase $=$ selection

\section{1}

Fig 6: Soar debugger window after phase selection command

one can trace the output that is useful for understanding episodic memory by the following command:

watch-epmem

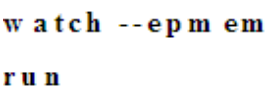

New episodic memory recorded for tim e 6 .

8: $==>S: S 17$ (state no-change)

New episodic memory recorded for tim e 7 .

9: $\quad==>S: S 21$ (state no-change)

New episodic memory recorded for tim e 8.

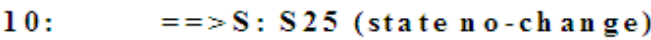

New episodic memory recorded for tim e 9.

11: $==>S: S 29$ (state no-change)

New episodic memory recorded for tim e 10 .

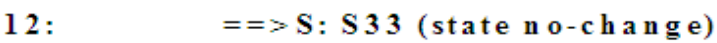

New episodic memory recorded for tim e 11 .

13 :

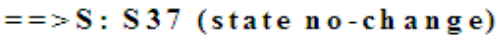

New episodic memory recorded for time 12 .

Fig 7: Soar debugger window to show the trace of episodic memory

\subsection{Agent Interaction}

Agents interact with episodic memory through special structures in working memory. Soar automatically creates an epmem link on each state and each epmem link has specialized substructure: a command link for agent initiated actions and $a$ result link for feedback from episodic memory, use the following command:

print --depth $5\langle s\rangle$ 
print -.d epth $5\langle s\rangle$

$(\$ 1$

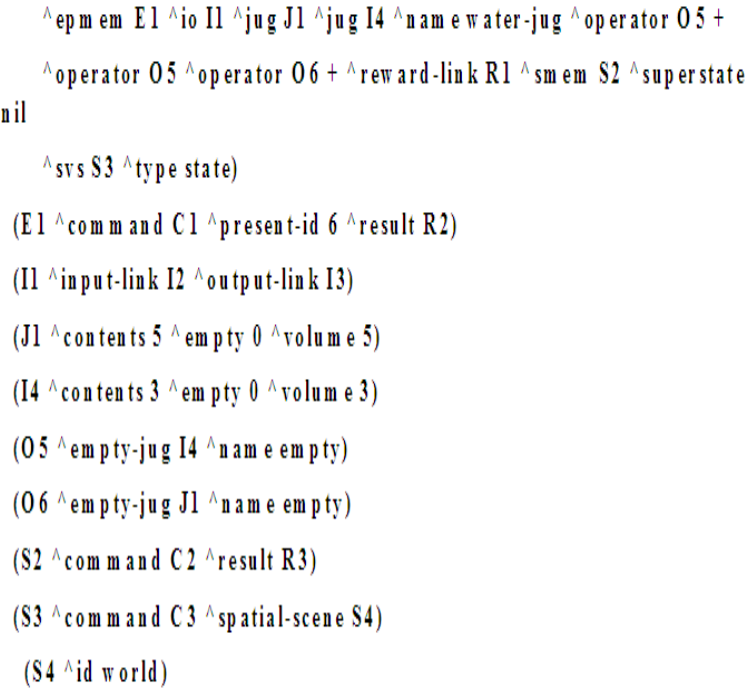

Fig 8: Soar debugger window to show the episodic memory link

\section{EXAMPLE}

Run the Soar Debugger and source the rules which are already part of the epmem-tutorial.Soar. Now execute the following commands:

$$
\begin{gathered}
\text {.epmem --set trigger } d c \\
\text { epmem --set learning on } \\
\text { watch-epmem }
\end{gathered}
$$

Click the "Step" button and then the "Run 1 -p" button.

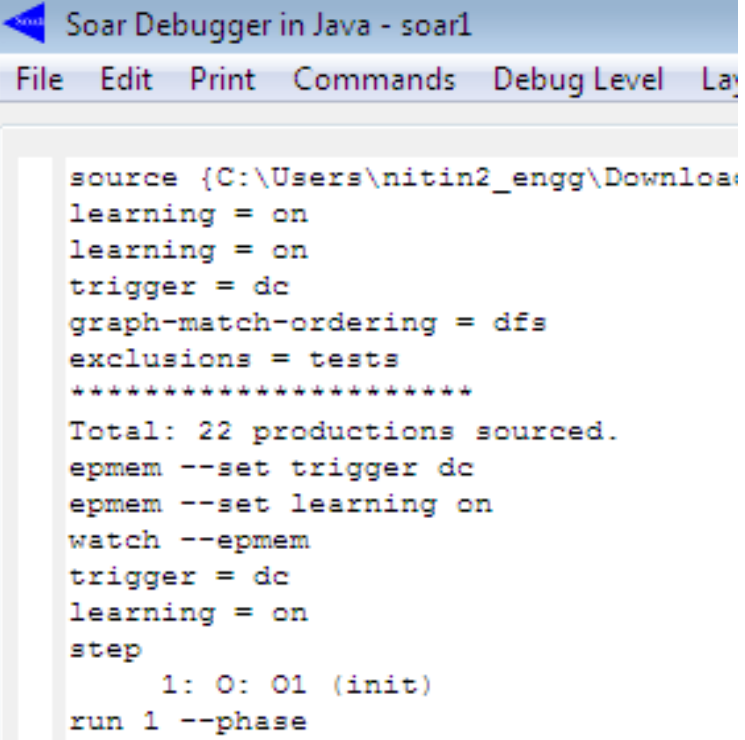

Fig 9: Soar debugger window to show the run by phases

To print the top State of working memory. Use the command: (print --depth $5 \quad$ sl).

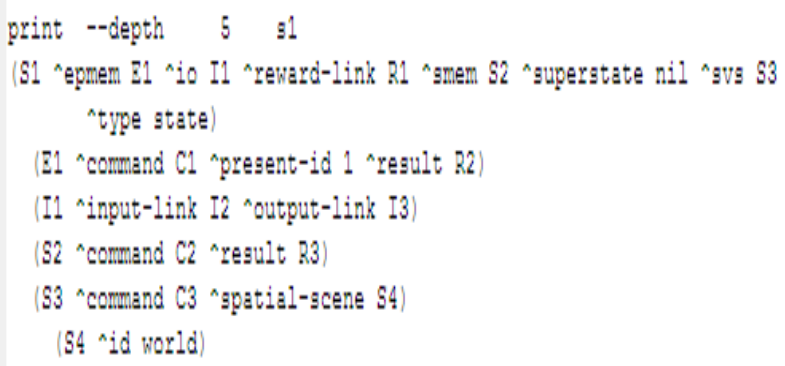

Fig 10: Soar debugger window with top state of working memory

To print the full contents of the episodic memory link we will use the command:

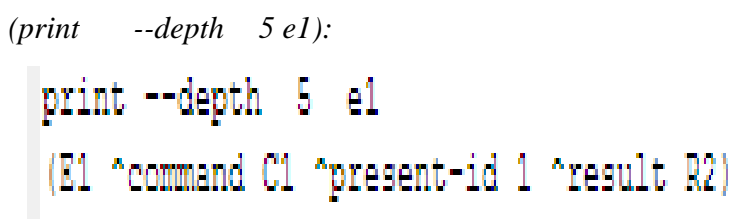

Fig 11: Soar debugger window showing the contents of episodic memory link

\subsection{Temporal Processing}

Another way the agent can gain access to episodes is by retrieving the episode that came temporally before/after the last episode that was retrieved. The syntax of these commands, respectively, are

$$
\begin{aligned}
&(<c m d>\wedge \text { previous }\langle i d\rangle) \\
& \text { And } \\
&(<c m d>\wedge \text { next }\langle i d>)
\end{aligned}
$$

Where <id> is any identifier. We will use following command:

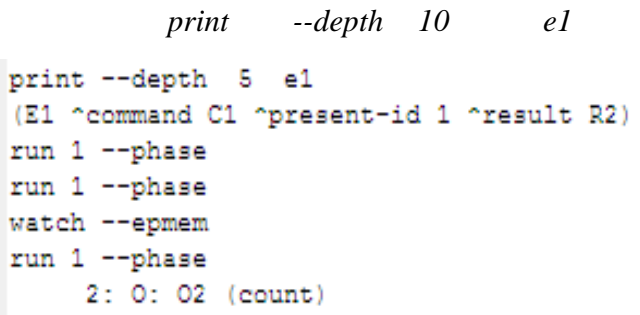

Fig 11: Soar debugger window showing temporal processing

The result structure has been cleaned of old structures and now shows that he command was successful and episode \#2 was retrieved.

\section{PERFORMANCE IMPROVEMENT}

Each element of an episodic memory as a Soar production. These productions fired (effectively retrieving the episodic memory) when a special retrieval operator was selected by the agent. This approach allowed us to focus on episode encoding while using Soar's existing storage and retrieval functionality. To work effectively, the recalled episode had to exactly match the cue. The most important stage in the episodic memory system is selecting the best match for a given memory cue. The manner in which memories are encoded and stored is important, but if the wrong episode is retrieved for an agent 
cue, agent decisions based upon that retrieval are suboptimal [8].

\section{CONCLUSION}

Episodic memory in cognitive architectures allows intelligent agents to query their past experiences to influence decision making. This approach allowed us to focus on episode encoding while using Soar's existing storage and retrieval functionality. To work effectively, the recalled episode had to exactly match the cue. Every time any state is reached for expansion in working memory it is checked with previous state stored in episodic memory, if it is repeated state or any of the state from solution path with the help of learning element and problem generator [5].

This effort established how soar agent learns to improve its performance. whole process is shown by implementing episodic memory in soar. This learning by experience helps the agent to improve its intelligence.

\section{REFERENCES}

[1] Laird, J. E, "The Soar Cognitive Architecture”,. MIT Press, Cambridge, MA, 20102.

[2] Bansal. N, Rajan. N., Srinivasan. S., March 30, 2013 , "Multi-Memory System: The Cognitive ArchitectureSOAR", NCACT-2013

[3] Andrew M. Nuxoll and John E. Laird, "Extending Cognitive Architecture with Episodic Memory", University of Michigan, Ann Arbor, MI 48109-2121
[4] Derbinsky. N., "Efficiently Implementing Episodic Memory in Soar", September 8, 2008.

[5] Laird, John \& Newell, Allen \& Rosenbloom, Paul, 1987, SOAR: An Architecture for General Intelligence. Artificial Intelligence. 33. 1-. 10.1016/00043702(87)90050-6.

[6] Endel Tulving, 2002, " EPISODIC MEMORY: From Mind to Brain", Annual Review of Psychology Vol. 53:1-25.

[7] Adrian Horzyk ; Janusz A. Starzyk; James Graham Dec. 2017," Integration of Semantic and Episodic Memories", IEEE Transactions on Neural Networks and Learning Systems, Volume: 28 , Issue: 12 ,.

[8] Nuxoll, A., "Enhancing Intelligent Agents with Episodic Memory", PhD Dissertation, University of Michigan, 2007.

[9] Tulving, E., "Elements of Episodic Memory", Oxford: Clarendon Press, 1983.

[10] Episodic memory available at "https://en.wikipedia.org/wiki/Episodic_memory".

[11] "Introduction to the Soar Cognitive Architecture", (PST $11)$, available http://acs.ist.psu.edu/misc/nottingham/pst/hungrythirsty/full.html 5/18, 2018. 